Copyright (C) 2020 University of Bucharest Printed in Romania. All rights reserved

ISSN print: $1224-5984$

ISSN online: $2248-3942$
Rom Biotechnol Lett. 2020; 25(1): 1216-1222

doi: $10.25083 / \mathrm{rbl} / 25.1 / 1216.1222$

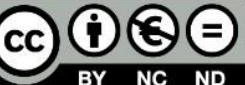

Received for publication, March, 2, 2019

Accepted, June, 3, 2019

Original paper

\title{
First vertebrates fluorescence protein UnaG: bacterial expression, purification and characterization
}

\author{
YAKUP ULUSU ${ }^{*}$
}

${ }^{1}$ Karamanoğlu Mehmetbey University, Faculty of Engineering, Department of Bioengineering, 70200 Karaman, Turkey

\begin{abstract}
Many fluorescence proteins and variants, including green fluorescent protein which was discovered in 1998, have been identified and used in scientific studies. In 2013, the first fluorescence protein $\mathrm{UnaG}$, originating from the vertebrate, was identified and its properties were demonstrated. In the present study, the UnaG protein was produced by fusion with TolAIII in Escherichia coli and its characterization was performed. In this study, the UnaG protein was purified by histidine affinity chromatography from Escherichia coli and, the presence and purity of the protein was demonstrated by sodium dodecyl sulphate polyacrylamide gel electrophoresis and western blotting; also, further characterization studies were performed by UV/ViS, Fluorescence and CD spectroscopy. According to Far-Uv CD results, the dominant secondary structure in the protein was determined as anti-parallel $\beta$-sheet. Thermal fluorescence studies have shown that the protein remains stable even at higher than room temperature. In the titration studies performed against $6,5 \mathrm{nM}$ protein, it was determined that the protein reached saturation at $11,67 \mathrm{nM}$ un-conjugated bilirubin concentration. It is possible to carry out different studies in the field of medicine and bioengineering by using UnaG, the first vertebrate source fluorescence protein that shows high affinity and specificity against un-conjugated bilirubin.
\end{abstract}

Keywords UnaG, fluorescent proteins, bilirubin.

To cite this article: ULUSU Y. First vertebrates fluorescence protein UnaG: bacterial expression, purification and characterization. Rom Biotechnol Lett. 2020; 25(1): 1216-1222. DOI: $10.25083 / \mathrm{rbl} / 25.1 / 1216.1222$

*Corresponding author: YAKUP ULUSU, Karamanoğlu Mehmetbey University, Faculty of Engineering, Department of Bioengineering, 70200 Karaman, Turkey

Telephone: +90 33822620 00-5034, Fax: +903382262023

E-mail: yakupulusu@kmu.edu.tr 


\section{Introduction}

The discovery of fluorescent proteins emerges as an important instrument in the progress of biomedical research. Green fluorescent protein (GFP) (1) and GFP-like proteins isolated from Aequorea victoria $(2,3)$, nearinfrared (NIR) fluorescence proteins have been extensively used in many experimental processes (4). Today, experimental biology with a newly developed fluorescence protein has been a revolution. In particular, no fluorescent protein was isolated from vertebrate living beings until 2013, while in 2013 Kumagai et al (5). identified UnaG, the first vertebrate originated fluorescent protein.

UnaGi; made from a freshwater eel (Anguilla japonica); it is one of the important traditional flavours of Japanese cuisine. These creatures travel thousands of kilometres in offshore to spawn throughout their life cycle. Larval travels over a few months to return to their original habitats in fresh water (6). This long migration cycle requires considerable adaptations to develop in skeletalmuscle physiology. These are particularly adaptations to the homeostasis of muscles and anaerobic oxidation metabolism during this non-stop swimming period. The first clue about this issue was highlighted by Hayashi and Toda in 2009 (7). This study was reported that the muscle fibres of A. japonica were fluorescence. In 2013, Kumagai intensified their work on the source of this fluorescence and identified the amino acid and related gene sequence by identifying the strong fluorescence-specific UnaG protein that could be induced with un-conjugated bilirubin for the first time.

Bilirubin emerges as a yellow product of normal haemoglobin destruction (especially the destruction of old red blood cells). Bilirubin is physiologically converted to water-soluble form in the liver by combining with glucuronic acid in the metabolism and is excreted from the body especially by bile and urine. However, in some cases the blood level of bilirubin continues to rise (hyperbilirubinemia), and consequently the passing to the brain tissue can be observed. This can result in a condition called kernicterus. For this reason, monitoring of bilirubin levels is extremely important especially in new-born infants.

UnaG is a protein composed of 139 amino acids synthesized in the Japanese freshwater eel Anguilla japonica muscle fibrils, and it is found among its fatty acid binding proteins. Between Human Fatty Acid Binding Proteins (HFABP) and UnaG alignment results reflect a homology (56\%) (8). Just like other fatty acid binding proteins (FABP), HoloUnaG has $\beta$-barrel structure formed by 10 antiparallel $\beta$-sheets, and this barrel carries two small $\alpha$-helix parts. The bilirubin binding site is located in the middle space of this barrel structure (5). During binding of bilirubin to ApoUnaG to form HoloUnaG, the exovinyl di-pirrhinone moiety is buried in the middle space of the protein in the $\beta$-barrel structure, and then the carboxylate groups and all the bilirubin are added to the protein via many hydrogen bonds. Bilirubin forms a number of hydrogen bonds with UnaG through water molecules, which results in the formation of a chromophore region that emits green fluorescence. This interaction is similar to other FABP-ligand complexes.

As noted above, when the observation of the fluorescence emission in the muscles of the Japanese freshwater eel is compared with other known fluorescence proteins has led to the discovery and characterization of the UnaG protein obtained from a completely different biological source and having a different fluorescence mechanism. It is obvious that UnaG is the first fluorescence protein from vertebrate living beings, as well as much more advanced advantages and potency of medical-industrial application compared to other proteins obtained from lower-minded creatures. UnaG is also the first fluorescence protein activated by a natural ligand (bilirubin) and this property gives the potential to be used in much different applications of the protein.

In this study, the UnaG protein was produced as a fusion with the TolA-III in the E. coli pTolT heterologous protein expression system, purified by affinity chromatography and UV/ViS, Fluorescence and CD spectroscopy characterization tests were performed.

\section{Material and Methods}

\section{Expression of TolAIII-UnaG Fusion Protein in E. coli}

The DNA fragment encoding the UnaG (GenBank: AB763906.1) (purchased from Biomatik, USA) was cloned into the pTolT expression vector using two restriction enzymes (BamH I and Kpn I) and expression of the protein in E. coli was performed via this system (Figure 1) (9).

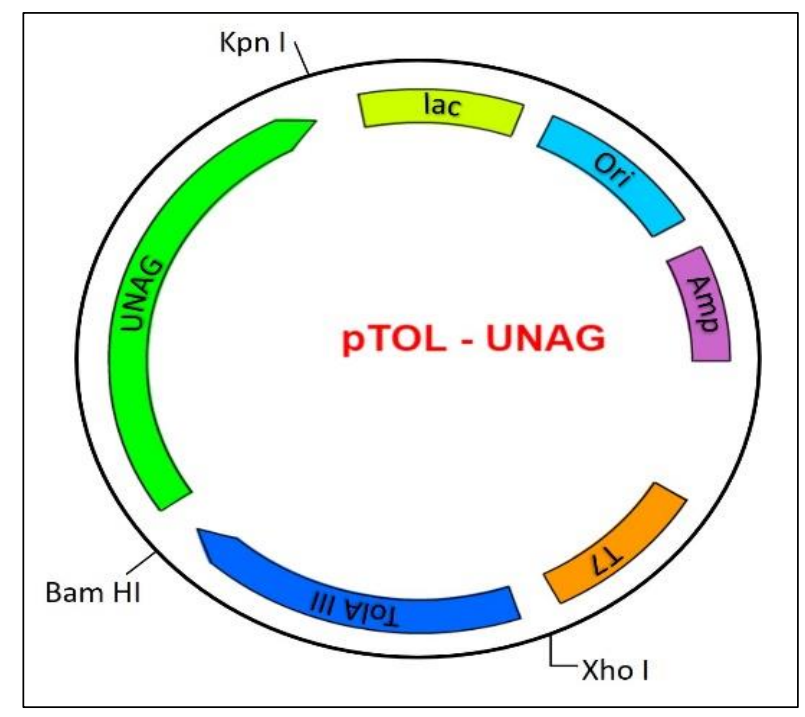

Figure 1. Plasmid map of the pTOL-UNAG construct to produce TolA-III and UnaG fusion.

This vector was named pTolT-UnaG and the cloning procedure was checked by verification PCR reaction, restriction validation analysis with appropriate restriction enzyme and DNA sequence analysis. Expression of the His-TolAIII-UnaG fusion protein in E. coli was performed 
after all these validation analysis results showed that the UnaG gene was correctly cloned into the pTolT expression system. The pTolT expression plasmid houses an operator gene region with the T7 promoter and the repression is removed by the addition of Isopropyl $\beta$-D-1-thiogalactopyranoside (IPTG) protein production is induced in this way. In this study, growing of the transformant E. coli cells (C41 strain) in LB medium were monitored by UV/Vis spectrophotometer (Shimadzu UV-1800 Spectrophotometer) at a wavelength of $600 \mathrm{~nm}$ and promoter induction was provided by adding IPTG in the log phase.

Following induction, the cells were grown at $35^{\circ} \mathrm{C}$ for additional 3 hours and then harvested and re-suspended with $20 \mathrm{mM}$ phosphate and $300 \mathrm{mM}$ sodium chloride $(\mathrm{NaCl})(\mathrm{pH} 8.00)$ buffer containing RNAse, DNAse, and protease inhibitors ( $1 \mathrm{mM}$ phenylmethylsulfonyl fluoride, $1 \mathrm{mM}$ benzamidine, $0.1 \mu \mathrm{M}$ aprotinin, $1 \mu \mathrm{M}$ leupeptin and $1 \mu \mathrm{M}$ pepstatin). Cells were sonicated with sonicator (Sonics Vibra-Cell) and supernatant containing soluble proteins was obtained by ultracentrifugation at $40.000 \mathrm{rpm}$ for 1 hour (Beckman Coulter Optima L-80 ultracentrifuge and $\mathrm{Ti} 45$ rotor). The GFP protein containing $6 \mathrm{X}$ His-Tag was purified by affinity chromatography by using a Ni-NTA agarose resin (Thermo Fisher Scientific). The supernatant obtained after the ultracentrifugation was applied to the center of the column and then the column was washed with $30 \mathrm{mM}$ imidazole-containing above mentioned buffer (pH 8.0) to elute non-specific binding. To elute the GFP proteins bound to the resin colon was washed with $300 \mathrm{mM}$ imidazole-containing buffer and the eluates were collected in fractions of $1 \mathrm{ml}$. In order to remove the imidazole used for elution during purification, each fraction was dialyzed against $5 \mathrm{~L}$ of the buffer throughout overnight at $4{ }^{\circ} \mathrm{C}$. The expression of His-TolAIII-UnaG is qualitatively analysed by sodium dodecyl sulphate polyacrylamide gel electrophoresis (SDS-PAGE) and Western Blotting by using Anti-His antibody (Sigma Aldrich).

\section{Circular Dichroism Analysis}

Circular Dichroism experiment was conducted using a Jasco 810 spectropolarimeter with $1 \mathrm{mg} \mathrm{mL}^{-1}$ TolAIIIUnaG protein in a $0.1 \mathrm{~cm}$ quartz glass cuvette (Hellma; 110-1-40 QS). Data were collected in triplicate for all conditions at a wavelength between $195-250 \mathrm{~nm}$, a data pitch of $0.2^{\circ} \mathrm{C}$, and a width of $2 \mathrm{~nm}$. Measurements were performed with both colourless fusion protein and as fluorescence with bilirubin.

\section{Fluorescence Spectroscopy Analysis}

Fluorescence spectroscopy analysis was performed using Varian Cary Eclipse Fluorescence Spectrophotometer. Excitation and emission values for the fusion protein purified and produced by this study for the first time were determined prior to the study of fluorescence spectroscopy with UNAG fluorescence protein and bilirubin. For the titration study, protein solution at the initial concentration of $6.5 \mathrm{nM}$ was used. Subsequently, the concentration of bilirubin was increased step by step until all the proteins were saturated with bilirubin. The fluorescence intensity corresponding to the bilirubin addition at each step was recorded. The addition of bilirubin continued to the point where the fluorescence intensity remained constant $(12.5 \mathrm{nM})$. At the same time, the intensity of fluorescence was also observed depending on the temperature. Fluorescence activity dependent on the temperature initiated at room temperature was maintained until $95^{\circ} \mathrm{C}$. The settings in Table 1 are used for fluorescence spectroscopy studies.

Table 1. Parameters used in fluorescence spectroscopy measurements.

\begin{tabular}{|c|c|}
\hline Excitation & $498 \mathrm{~nm}$ \\
\hline Excitation slit $(\mathrm{nm})$ & 20 \\
\hline Emission slit $(\mathrm{nm})$ & 20 \\
\hline Wavelength range & $500-550 \mathrm{~nm}$ \\
\hline Scan control & Slow \\
\hline PMT Detector Voltage & Medium \\
\hline
\end{tabular}

\section{Results and Discussion}

The observation of fluorescence illumination in the muscle cells of the Japanese fresh water eel (Anguilla japonica) has opened the door to the discovery of the UnaG, a novel protein that radiates with a different mechanism from all known fluorescent proteins. UnaG has the opportunity to find a place in many different areas in medical and experimental researches compared to other fluorescent proteins originating from lower organisms thanks to its advantages of obtaining vertebrates. In addition, UnaG is a protein that can be activated by a natural ligand and can play a key role in many research fields. In a study performed in 2013, (5) this ligand was found to be lipophilic heme metabolite un-conjugated bilirubin. Owing to the fact that, UnaG can generate fluorescence emission independent of oxygen, it is possible that studying with this protein even under the anaerobic conditions which are GFP-derived fluorescence proteins are inactive $(1,3)$.

Consequently, UnaG protein which contains all these characteristics was first produced by Kumagi et al, 2013 together with the GST tag, and its 3D structure was determined. In our study, UnaG protein was characterized as a fusion with TolAIII, an E.coli periplasmic protein. A chimeric structure for production has been preferable because the UnaG protein, which is expressed by a vertebrate (Anguilla japonica), may have a toxic effect for $E$. coli and is presumedly able to form the inclusion body. These possibilities were prevented by directing the UnaG to the periplasmic site of $E$. coli with TolA III protein. Moreover, the affinity of the GST tag used for the purification of UnaG expressed in the previous study (5) against to bilirubin (10) has caused the cleavage of the chimeric protein during characterization studies. In the present study, the TolAIII-UnaG fusion protein was characterized without cleaving.

\section{Expression and Purification of TolAIII-UnaG Fusion}

Details on the construction of pTOL-UnaG plasmid are given in Figure 1. This vector is particularly used in expression studies of toxic proteins and allows for strict control of protein production through the $\mathrm{T} 7$ promoter 
$(11,12)$. Our construct also included an N-terminal polyhistidine tag (6X histidines) for simple and efficient protein purification (Figure 2). DNA sequencing of this constructed plasmid confirmed that the desired inserts were successfully cloned into the pTOLT expression vector and also that the fusion constructs were in the correct reading frame. Transformation of this new construct into Escherichia coli $\mathrm{C} 41$ cells was done by heat shock and protein expression was performed. Proteins containing the histidine affinity tag were able to bind to the Ni-NTA agarose resin with a greater affinity than other $E$. coli proteins. Proteins that were not specifically bound were washed through the column without affecting the binding of His-tagged fusion protein. Elution of the fusion protein from the column was achieved by imidazole, which competes with the His tag for interaction with the Ni-NTA resin. Eluted samples were analysed on SDS-PAGE and western blot and showed a strong protein band with a molecular weight of around $27 \mathrm{kDa}$. The calculated molecular weight of the fusion protein using the ProtParam tool was $27,3 \mathrm{kDa}$, which is very close to the experimental molecular weight. The extinction coefficient fusion at $280 \mathrm{~nm}$ is 24,535 and its theoretical pI is 8,63 (http://expasy.proteome.org.au/cgibin/protparam).

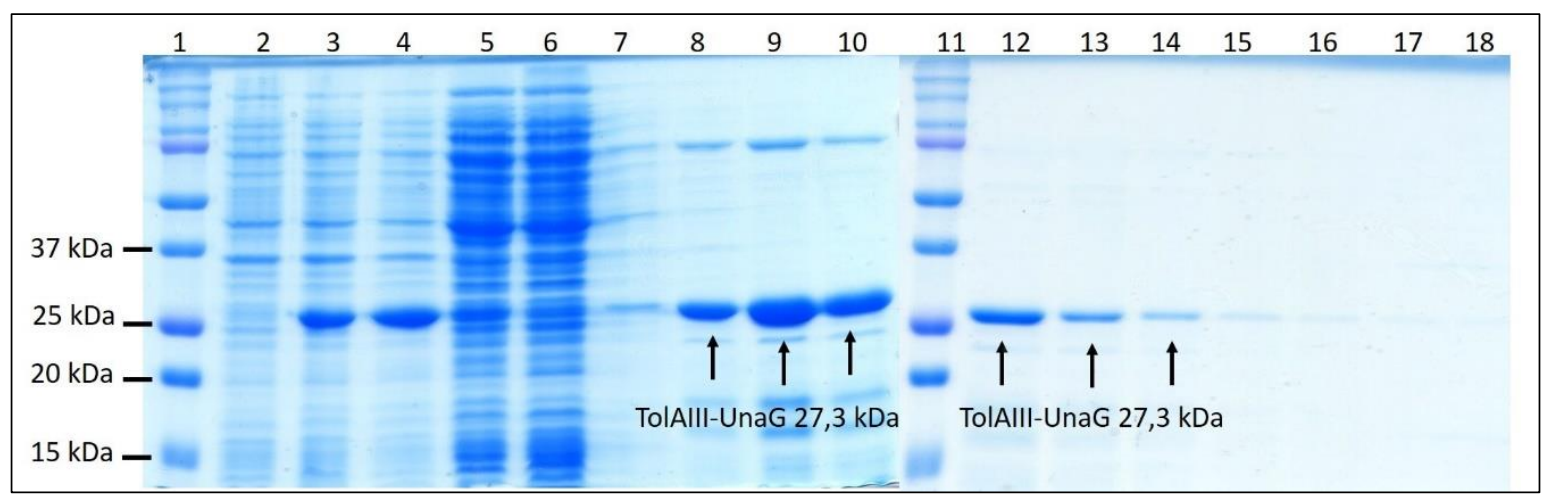

Figure 2. $12 \%$ SDS-PAGE (From c41 cells); 1) Marker (Biorad Precision Plus); 2) Un-induced cell pellet; 3) 1 hour later after IPTG induction; 4) 3 hour later after IPTG induction; 5) Load (Before Ni-NTA Agarose column); 6) Flow-through (after Ni-NTA Agarose column); 7) Wash; 8-18) Elutions.

\section{Western Blot Analysis}

The presence of the TolAIII-UnaG fusion is shown with the western blotting against the 6XHis tag found in the N-terminal in the purified protein (Figure 2). For this procedure, begin with an SDS-PAGE was carried out using eluants, followed by transfer to a 0,2-micron nitrocellulose membrane without gel staining. The transfer of the proteins using Mini-PROTEAN Tetra Cell, Mini TransBlot Module system to the membrane was controlled by the Ponceau S solution. 6x His Monoclonal Antibody from Mouse (Neo-Biotech, France) was used as primary antibody and Anti-Mouse IgG -Peroxsidase (Sigma, USA) was used as secondary antibody.

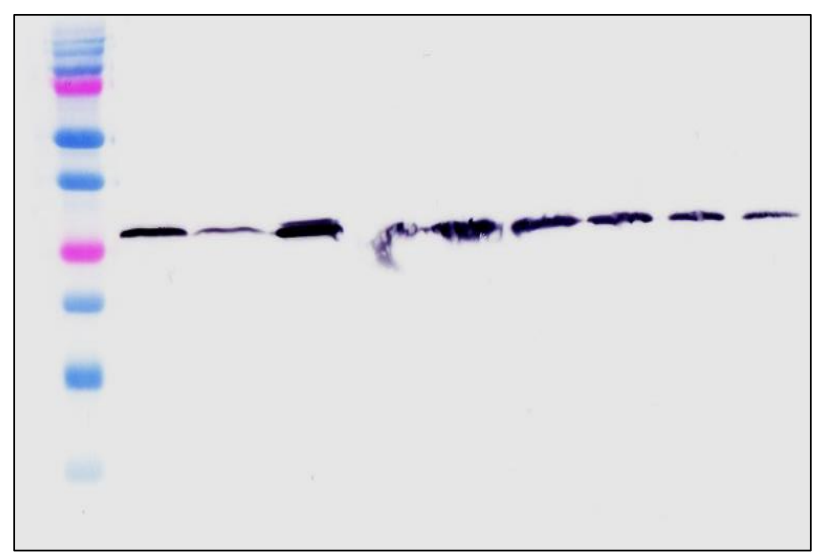

Figure 3. Western Blot Analysis of purified TolAIII-UnaG Protein. 1) Marker (Biorad Precision Plus). 2) Purified His Tagged TolAIII-UnaG Elutions.

\section{UV/Vis Absorbance of apo/holoUnaG}

The UV/VIS absorbance of TolAIII-UnaG produced and purified in the E. coli pTOLT expression system as a chimeric was analysed by Shimadzu UV1800 spectro- photometer. As seen in the obtained UV absorbance graph in Figure 4, both apoUnaG and holoUnaG showed maximum absorption at $280 \mathrm{~nm}$ due to the amino acid residues having the aromatic side group they contain. 
The holoUnaG (BLR added) showed the second absorbance at $510 \mathrm{~nm}$ corresponding to the green region of the light spectrum with its absorbance at $280 \mathrm{~nm}$. As it can be understood from the result presented in Figure 4, with the addition of BLR, apoUnaG was transformed into holo $\mathrm{UnaG}$ and the formation of fluorophore was realized.

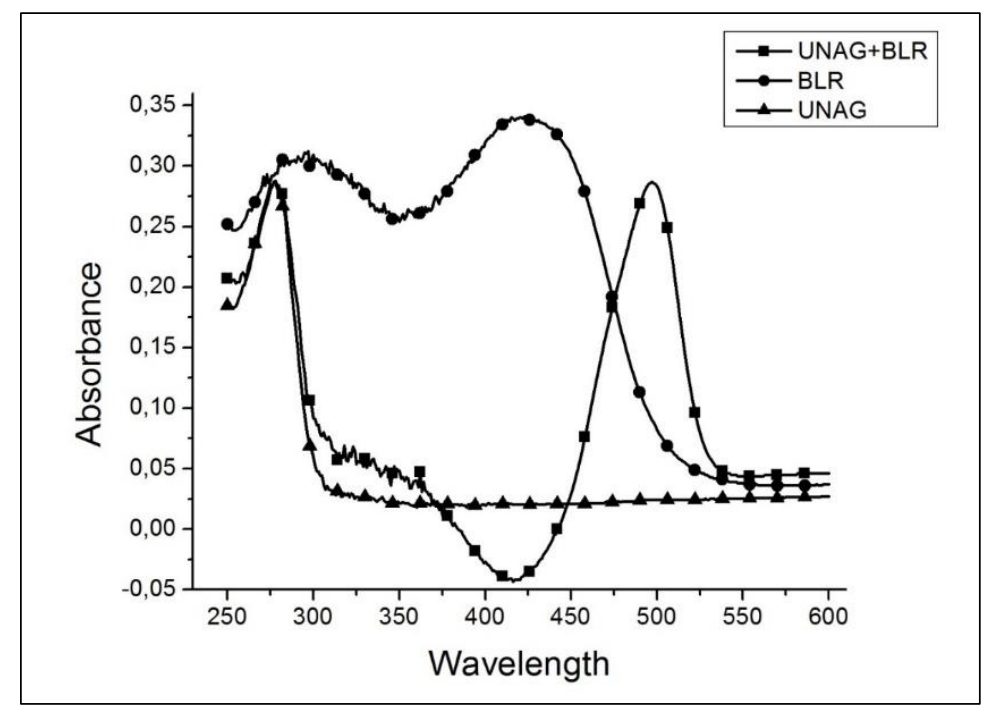

Figure 4. UV/Vis absorbance of apo/holoUnaG

\section{Far UV CD analysis of apo/holoUnaG}

Circular dichroism (CD) is one of the methods frequently used by researchers as a fast and easy method to analyse the structure of proteins in solution in recent years. In order to receive a signal from the spectropolarimeter, it is necessary to locate at least one chiral center within the molecule. This can occur in the case that the $\mathrm{C}$ atom binds with 4 different groups, presence of disulfide crosslinks stabilizing the 3-dimensional conformation of proteins, covalent bonding of the molecule to another molecule with a chiral center or has asymmetric environmental conditions (13).

In proteins, usually peptide bonds are used as chromophores that form the Far UV CD signal. As a result, CD signals from a protein molecule may vary depending on its environmental conditions or the ligand to which it binds. In fact, the change in CD signal can be considered as an indicator of protein ligand interaction. As can be seen in Figure 5, BLR interacted with apoUnaG, leading to the occurrence of holoUnaG, resulting in a change in the 3D structure of the protein. This shift in FarUV CD spectra can be considered as a sign of BLR binding with TolAIII-UnaG protein. As can be understood from the far UV CD signal, the protein that was expressed and purified as a fusion with TolAIII in E. coli gained the 3-dimensional conformation. The CD signal indicates that the secondary structure predominating in the protein is antiparallel $\beta$-sheet.

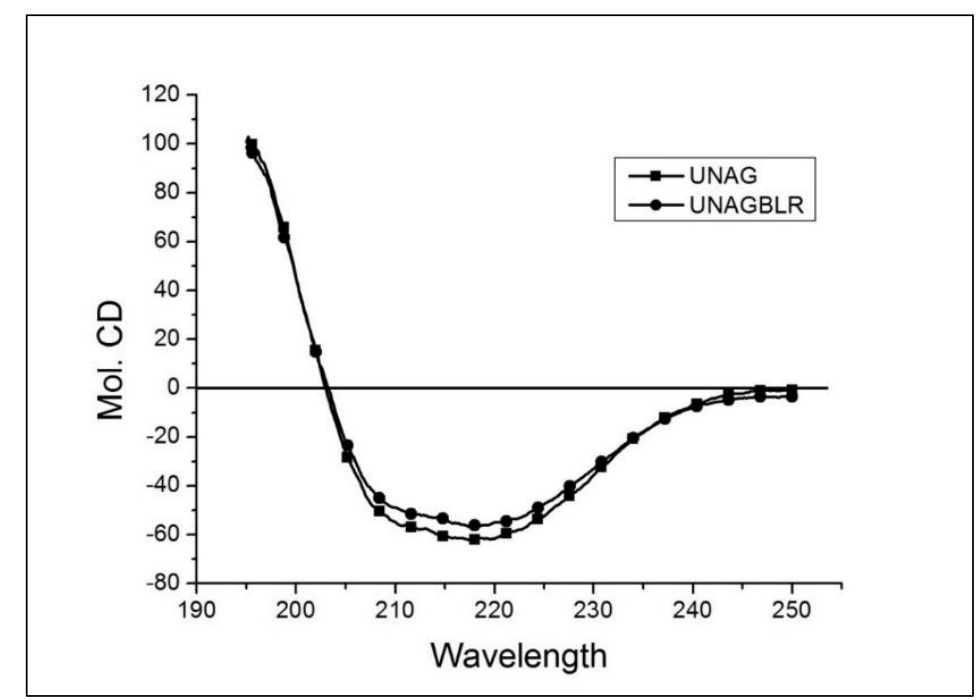

Figure 5. Far-UV CD spectrum of apo/holoUnaG 


\section{Fluorescence Analysis of TolAIII-UnaG Fusion Protein}

The excitation and emission points, the temperaturedependent changes of fluorescence emission (thermal unfolding studies of the protein) and the changes in fluorescence intensity with varying concentrations of bilirubin of the protein produced in the E. coli PToLT expression system were analysed by the fluorescence spectrophotometer. The chimeric protein showed maximum excitation and emission at 498 and $527 \mathrm{~nm}$ respectively (Figure 6a). The TolAIII-UnaG fusion developed in this study has showed similarity with the wild type reported in the literature (5). According to the temperature dependent fluorescence emissions measured with HoloUnag, it was determined that at least half of the protein produced in this study was stable up to $45^{\circ} \mathrm{C}$ and maintained its fluorophore structure. In addition, it was determined that some part of the protein still retained the 3-D structure around $60^{\circ} \mathrm{C}$ (Figure 6b). In this sense, TolAIII-UnaG fusion which shows maximum fluorescence emission in the room temperature conditions has a high chance of being used in a bilirubin biosensor which is likely to be carried out in future studies. This version of UnaG opens the way to be a preferable molecule in terms of both storage conditions and a shelf life of a sensor that can be developed in the future.

The affinity of the TolAII-UnaG fusion against un-conjugated bilirubin (UC-BLR) was determined by the fluorometric method, and the initial concentration of the protein was to $6.5 \mathrm{nM}$. During titration studies all of the apoUnaG proteins present in the solution were coupled to un-conjugated bilirubin to achieve maximum fluorescence emission at the around $11.67 \mathrm{nM}$ bilirubin concentration. Despite the increase in the concentration of bilirubin after this step, no change in fluorescence emission showed that all apo-UnaGs in the solution were completely converted into halo-UnaG and reached to saturation (Figure 6c).

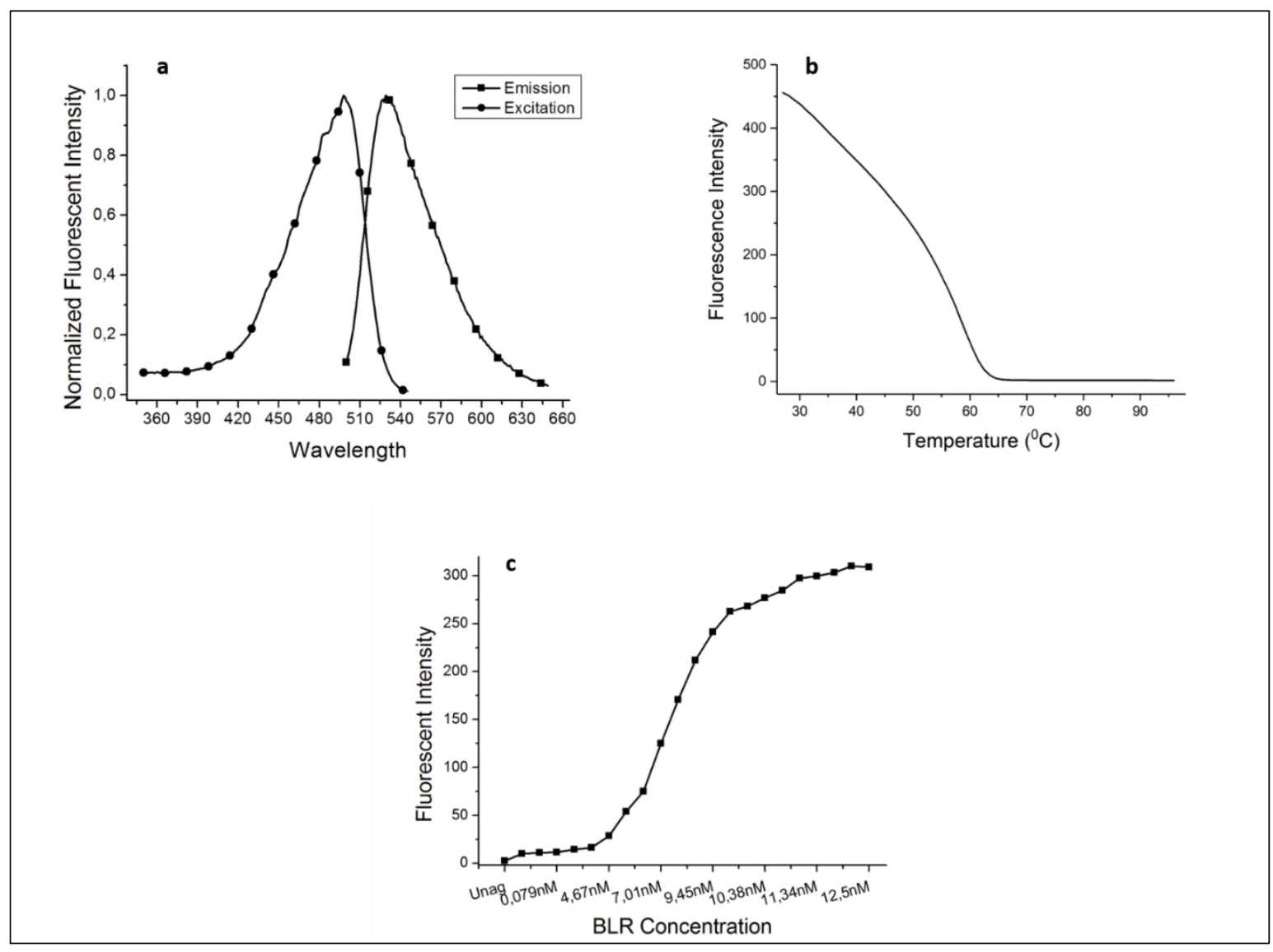

Figure 6. a. Excitation and emission spectrum of Holo-UnaG; b. Changes of the fluorescence intensity of the Holo-UnaG by temperature; c. Changes of the fluorescence intensity of the Holo-UnaG by BLR concentration.

\section{Conclusions}

As a result, in this study UnaG protein belonging A. japonica was purified by expressing it in fusion with TolAII in E. coli prokaryotic expression system. The fusion protein was purified and characterized by affinity chromatography using 6X His Tag found in the PtolT expression system. The pure protein was indicated by Western blotting and the secondary structure of the protein was tried to be explained by circular dichroism spectropolarimeter. As is 
evident from the far UV CD spectra, the protein has a threedimensional conformation dominated by the antiparallel $\beta$-sheet structure. In addition, the Un-conjugated bilirubin is attached to the protein, which is understood by a slight shift in the Far-UV CD spectrum. On the other hand, fluorescence studies have shown that the obtained protein from this study has affinity for unconjugated bilirubin at the nanomolar level and holoUnaG can remain stable even at temperatures above room temperature. In the further studies un-conjugated bilirubin biosensor can be developed by a number of protein engineering applications on TolAIII-UnaG fusion developed with this study.

\section{Acknowledgments}

Preparation of the gene construct used in the study, I would like to express my gratitude to Prof. Dr. Isa GÖKÇE and thanks to Prof. Dr. Jeremy LAKEY for the opportunity to work in his laboratory for performing the $\mathrm{CD}$ analysis.

\section{References}

1. TSIEN, Y., R., The Green Fluorescent Protein, Annu. Rev. Biochem., 1998, vol. 67, pp. 509-544.

2. CHUDAKOV, D.M., MATZ, M.V., LUKYANOV, S., LUKYANOV, K.A., Fluorescent proteins and their applications in imaging living cells and tissues, Physiol. Rev., 2010, vol. 90, pp. 1103-1163.

3. MIYAWAKI, A., SHCHERBAKOVA, D.M., VERKHUSHA, V.V., Red fluorescent proteins: chromophore formation and cellular applications, Curr. Opin. Struct. Biol., 2012, vol. 22, pp. 679-688.

4. PIATKEVICH, K.D., SUBACH, F.V., VERKHUSHA, V.V., Engineering of bacterial phytochromes for near-infrared imaging, sensing, and light-control in mammals, Chem. Soc. Rev., 2013, vol. 42, pp. 34413452.

5. KUMAGAI, A., ANDO. R., MIYATAKE. H., GREIMEL. P., KOBAYASHI. T., HIRABAYASHI. Y., SHIMOGORI. T, MIYAWAKI. A., A BilirubinInducible Fluorescent Protein from Eel Muscle, Cell., 2013, vol. 153, pp. 1602-1611.

6. TSUKAMOTO, K., Oceanic biology: spawning of eels near a seamount, Nature., 2006, vol. 439, p. 929.

7. HAYASHI, S., TODA, Y., A novel fluorescent protein purified from eel muscle, Fish. Sci., 2009, vol. 75, pp. 1461-1469.

8. ZIMMERMAN, A.W., VEERKAMP, J.H., New insights into the structure and function of fatty acidbinding proteins, Cell. Mol. Life Sci., 2002, vol. 59, pp. 1096-1116.

9. ULUSU, Y., BILGIN ŞENTÜRK, S., KUDUĞ, H., GÖKÇE, İ., Expression, purification, and characterization of bovine chymosin enzyme using an inducible pTOLT system, Preparative Biochemistry and Biotechnology, 2016, vol. 46, pp. 596-601.

10. ARIAS, I.M., OHMI, N., BHARGAVA, M., LISTOWSKY, I., Ligandin: an adventure in liverland, Mol. Cell. Biochem., 1980, vol. 29, pp. 71-80.

11. STUDIER, F.W., MOffATT, B.A., Use of bacteriophage T7 RNA polymerase to direct selective highlevel expression of cloned genes, J Mol Biol., 1986, vol. 189, pp. 113-130.

12. CHEN, X., HUANG, Z., ZHOU, B., WANG, H., JIA, G., QIAO, J., Expression and purifcation of porcine Akirin2 in Escherichia coli, Turk J Biol., 2014, vol. 38, pp. 339-345.

13. KELLY, S.M., JESS, J.T., PRICE, N.C., How to study proteins by circular dichroism, Biochimica et Biophysica Acta, 2005, vol. 1751, pp. 119-139. 